\title{
A rare case of dysphagia resulting from complete oesophageal obstruction
}

\author{
Mateusz Jagielski, Jacek Piątkowski, Marek Jackowski \\ Department of General, Gastroenterological and Oncological Surgery, Collegium Medicum Nicolaus Copernicus University, \\ Torun, Poland
}

Gastroenterology Rev 2020; 15 (4): 354-355

DOI: https://doi.org/10.5114/pg.2020.95873

Address for correspondence: Ass. Prof. Mateusz Jagielski MD, PhD, Department of General, Gastroenterological and Oncological Surgery, Collegium Medicum Nicolaus Copernicus University, 53-59 Sw. Jozefa St, 87-100 Torun, Poland, phone: +48 566793199 ,

e-mail: matjagiel@gmail.com

A 71-year-old woman was admitted to our department in December 2018 showing symptoms of dysphagia. The patient reported swallowing difficulties, which had increased over the last 6 months. She was unable to swallow solid food 1 week before admission and unable to consume any liquids for 2 days. Over the previous 6 months, she had lost $30 \mathrm{~kg}$ of bodyweight. In February 2017, laparoscopic Nissen-Rossetti fundoplication and mesh hiatoplasty were performed due to symptomatic hiatal hernia (Figure 1). A gastroscopy was performed on the day of admission (December 2018), which showed oesophageal obstruction due to complete mesh migration into the lumen of the oesophagus in the oesophagogastric junction area (Figure 2). The patient was qualified for surgical treatment based on the clinical picture and results of imaging examinations. A covered oesophageal perforation with mesh migration was observed intraoperatively. Partial resection of the oesophagus and resection of the cardia with oesophagogastric anastomosis and extraction of the mesh were performed using a combined endoscopic and laparoscopic approach (Figure 3). Re-plasty of the hiatal hernia was not performed because the hiatus had been reduced in size by the previous operation. Total parenteral nutrition was gradually replaced with an oral diet during the postoperative period. No complications occurred during surgery or the postoperative period. The patient was discharged on postoperative day 7 in a good general condition without any ailments and showing good tolerance to an oral diet. A control gastroscopy in June 2019 showed a correct endoscopic picture of oesophagogastric anastomosis. No other pathological changes were diagnosed in the upper gastrointestinal tract.

Laparoscopic repair with synthetic mesh implantation is a standard procedure in the surgical treatment of symptomatic hiatal hernia [1, 2]. After reviewing the current literature, we found only a few studies that described complete mesh migration into the lumen of the oesophagus that resulted as a postoperative complication of hiatal hernia [1-5]. Moreover, none of these studies described any cases of complete oesophageal obstruction caused by mesh migration that resulted in dysphagia [1-5]. Furthermore, the pathomechanism underlying this type of complication remains unexplained [1-5]. However, we are unable to clarify why patients with oesophageal perforation caused by mesh migration are generally in good condition and show no symptoms other than dysphagia [1-5]. The current report presents a very rare case of dysphagia, which resulted as a late complication of surgical treatment, occurring 12 months after the procedure. In the manuscript an innovatory procedure was presented: a combination of endoscopic and laparoscopic treatment of the complication.

\section{Conflict of interest}

The authors declare no conflict of interest. 


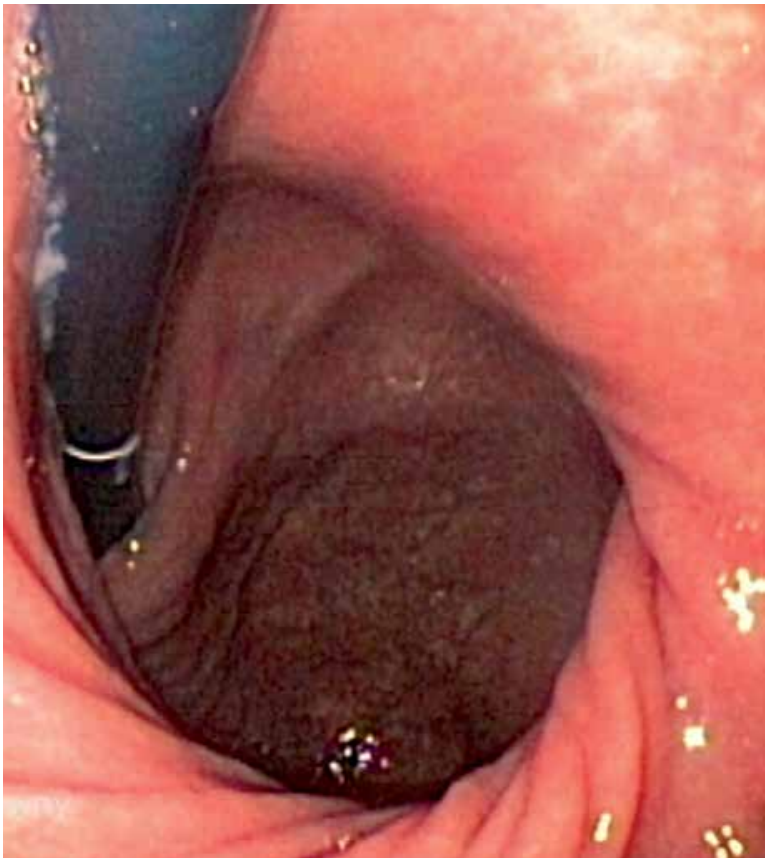

Figure 1. Gastroscopic image in inversion. Extensive hiatal hernia (black arrows) before surgery is visible

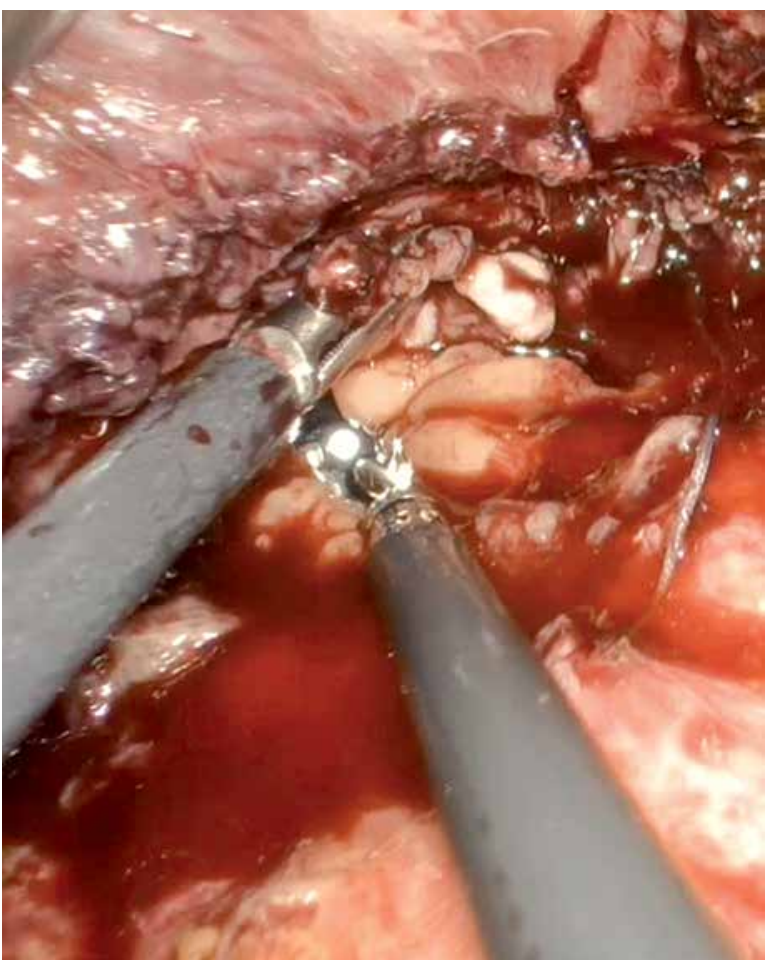

Figure 3. Intraoperative images taken during laparoscopy. An endoscope in the lumen of the oesophagus - a view from the side of the peritoneal cavity through the point of oesophageal perforation

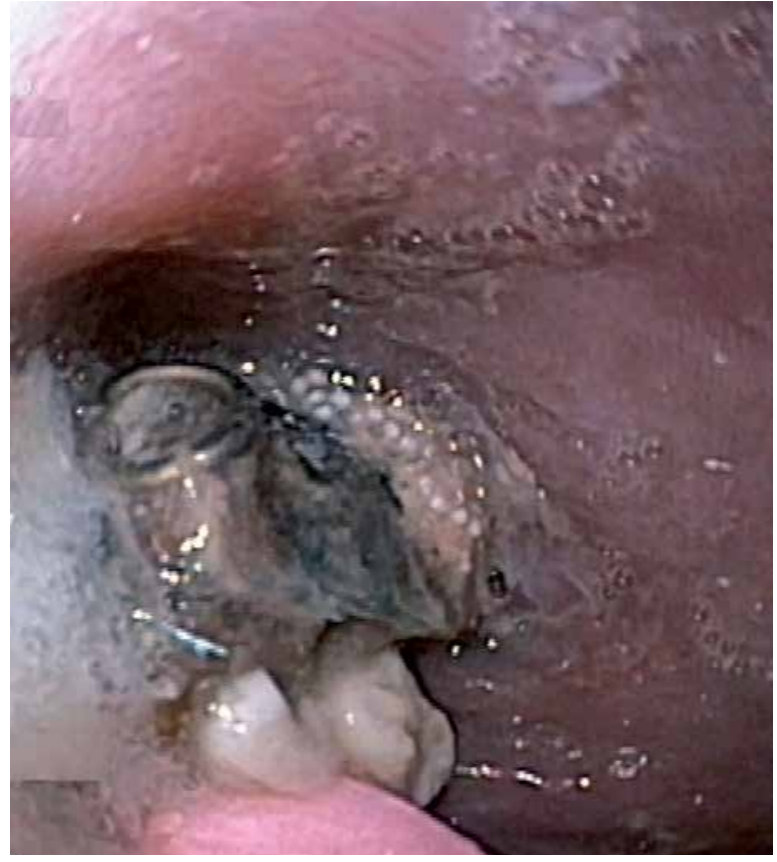

Figure 2. Endoscopic picture. Complete mesh migration (white arrows) is visible in the oesophagogastric junction area

\section{References}

1. Tam V, Winger DG, Nason KS. A systematic review and meta-analysis of mesh vs suture cruroplasty in laparoscopic large hiatal hernia repair. Am J Surg 2016; 211: 226-38.

2. Antoniou SA, Müller-Stich BP, Antoniou GA, et al. Laparoscopic augmentation of the diaphragmatic hiatus with biologic mesh versus suture repair: a systematic review and meta-analysis. Langenbecks Arch Surg 2015; 400: 577-83.

3. Oguri Y, Cho H, Yajima K, et al. Case of complete mesh migration into the stomach after mesh hiatoplasty for a hiatal hernia. Asian J Endosc Surg 2018; 11: 395-8.

4. Griffith PS, Valenti V, Qurashi K, et al. Rejection of Goretex mesh used in prosthetic cruroplasty: a case series. Int J Surg 2008; 6: 106-9.

5. Stadlhuber RJ, Sherif AE, Mittal SK, et al. Mesh complications after prosthetic reinforcement of hiatal closure: a 28-case series. Surg Endosc 2009; 23: 1219-26.

Received: 17.03 .2020

Accepted: 4.05 .2020 